\title{
Analgesic Effect of Toll-like Receptor 4 Antagonistic Peptide 2 on Mechanical Allodynia Induced with Spinal Nerve Ligation in Rats
}

\author{
Yuhua Yin ${ }^{1,2}$, Hyewon Park ${ }^{1,2}$, Sun Yeul Lee ${ }^{3}$, Won-hyung Lee ${ }^{3}$, Hee-Jung Song ${ }^{4}$, \\ Jinhyun $\mathrm{Kim}^{3}$, Dong Woon Kim ${ }^{1,2 *}$ and Jinpyo Hong ${ }^{2 *}$ \\ ${ }^{1}$ Department of Medical Science, Chungnam National University School of Medicine, Daejeon 35015, ${ }^{2}$ Department of \\ Anatomy, Brain Research Institute, Chungnam National University School of Medicine, Daejeon 35015, ${ }^{3}$ Department \\ of Anesthesia and Pain Medicine, Chungnam National University Hospital, Daejeon 35015, ${ }^{4}$ Department of Neurology, \\ Chungnam National University Hospital, Daejeon 35015, ${ }^{5}$ Department of Internal Medicine, Chungnam National University \\ Hospital, Daejeon 35015, Korea
}

\begin{abstract}
Neuroinflammation is one of the key mechanisms of neuropathic pain, which is primarily mediated by the Toll-like receptor 4 (TLR4) signaling pathways in microglia. Therefore, TLR4 may be a reasonable target for treatment of neuropathic pain. Here, we examined the analgesic effect of TLR4 antagonistic peptide 2 (TAP2) on neuropathic pain induced by spinal nerve ligation in rats. When lipopolysaccharide (LPS)-stimulated BV2 microglia cells were treated with TAP2 $(10 \mu \mathrm{M})$, the mRNA levels of proinflammatory mediators, such as tumor necrosis factor (TNF)- $\alpha$, interleukin (IL)-1 $\beta$, IL-6, cyclooxygenase (COX)-2, and inducible nitric oxide synthase (iNOS), were markedly decreased by $54-83 \%$ as determined by quantitative PCR (qPCR) analysis. Furthermore, when TAP2 (25 nmol in $20 \mu \mathrm{L}$ PBS) was intrathecally administered to the spinal nerve ligation-induced rats on day 3 after surgery, the mechanical allodynia was markedly decreased for approximately 2 weeks in von Frey filament tests, with a reduction in microglial activation. On immunohistochemical and qPCR analyses, both the level of reactive oxygen species and the gene expression of the proinflammatory mediators, such as TNF- $\alpha$, IL-1 $\beta$, IL-6, COX-2, and iNOS, were significantly decreased in the ipsilateral spinal dorsal horn. Finally, the analgesic effect of TAP2 was reproduced in rats with monoiodoacetate-induced osteoarthritic pain. The findings of the present study suggest that TAP2 efficiently mitigates neuropathic pain behavior by suppressing microglial activation, followed by downregulation of neuropathic pain-related factors, such as reactive oxygen species and proinflammatory molecules. Therefore, it may be useful as a new analgesic for treatment of neuropathic pain.
\end{abstract}

Key words: Neuropathic pain, Toll-like receptor 4, Analgesics, Microglia

Received January 31,2019, Revised May 11,2019, Accepted May 28, 2019

* To whom correspondence should be addressed.

Jinpyo Hong, TEL: 82-42-580-8209, FAX: 82-42-580-4800, e-mail: biohjp@gmail.com

Dong Woon Kim, TEL: 82-42-580-8207, FAX: 82-42-580-4800, e-mail: visnu528@cnu.ac.kr

Jinpyo Hong author's current address: Department of Neuroscience and Physiology, and Dental Research Institute, School of Dentistry, Seoul National University, Seoul 08826, Korea. Tel: +82-2-880-2314, Fax: +82-2-880-2314, e-mail: biohjp92@snu.ac.kr 


\section{INTRODUCTION}

Several neurotransmitters, including glutamate, adenosine triphosphate (ATP), and substance $\mathrm{P}$, are released from the nerve terminals of primary afferent neurons when peripheral nerves are injured by physical and/or inflammatory stress in diseases, such as diabetes and cancer. These molecules bind to their corresponding receptors on spinal cord neurons and then transmit pain signals to the brain via spinothalamic pathways by depolarizing the neurons. These molecules also stimulate surrounding spinal microglia and consolidate the pain by inducing secretion of proinflammatory mediators and strengthening the interactions between neurons and microglia [1]. In addition, several endogenous molecules, such as heat shock proteins, high mobility group box 1 (HMGB1), hyaluronan fragments, and heparin sulfate, are released from the injured neurons and induce activated microglia to produce inflammatory molecules, such as tumor necrosis factor (TNF)- $\alpha$, interleukin (IL)-1 $\beta$, IL-6, nitrogen oxide (NO), and prostaglandins, via Toll-like receptor (TLR)-mediated signaling pathways [2,3]. These substances may further solidify chronic pain by central sensitization in the spinal cord.

TLR4 is a member of the TLR family that recognizes pathogenassociated molecular patterns expressed on infectious agents, such as bacteria, viruses, and fungi. TLR4 binds to lipopolysaccharide (LPS) on most gram-negative bacteria, leading to activation of the innate immune system via an intracellular signaling cascade of nuclear factor (NF)- $\mathrm{kB}$ and interferon regulatory factor (IRF) 3 [4]. In addition, TLR4 recognizes a number of endogenous molecules (damage-associated molecular patterns), such as low-density lipoprotein, beta-defensin, fibrinogen, HMGB1, and heat shock proteins, released from the damaged tissue in vivo [5]. In the context of neuropathic pain, TLR4 is upregulated exclusively on the surface of microglia in the spinal cord in animal models of neuropathic pain [6]. Upon peripheral nerve injury, endogenous TLR4 ligands such as extracellular matrix components and HMGB1 released from injured neurons may excite spinal microglia via TLR4 to secrete inflammatory molecules further complicating the condition $[7,8]$. These ligands can also exacerbate pain because TLR4 expressed on the surface of sensory neurons (small diameter, C-fibers) was suggested to be involved in pain conduction [9]. In addition, TLR4-mediated microglial activation led to neuropathic pain by impaired autophagic flux in neurons in chronic construction injury (CCI)-induced mice [10]. In animal models of neuropathic pain with spinal nerve transection and CCI, mechanical allodynia was markedly decreased in TLR4 knockout mice compared with wild-type controls $[10,11]$. Therefore, blocking TLR4 signaling transduction using specific TLR4 antagonists may effectively re- lieve chronic pain.

There are several types of TLR4 antagonists/blockers, including Berberine, Sparstolonin B, Eritoran, TAK-242, IAXO102, FP7, CRX-526, FP-1, (+)-naloxone, and TLR4-C34 [12]. These comprise natural compounds, synthetic LPS analogues, and small molecules used to treat TLR4-mediated inflammatory diseases, such as sepsis, lethal influenza infection, and inflammatory bowel disease. For example, in response to LPS, Eritoran was shown to prevent the production of inflammatory mediators by competitively blocking the binding of LPS to TLR4/MD2 with consequent inhibition of the NF- $\mathrm{kB}$ signaling cascade [13]. Moreover, these molecules may attenuate neuropathic pain by blocking the TLR4mediated signaling pathway, because this pathway is critical for the initiation and maintenance of chronic pain [7]. In addition, subcutaneous administration of (+)-naloxone at a high dose of $10 \mathrm{mg} / \mathrm{kg}$ reversed chronic neuropathic pain in rats with chronic construction injury and spinal nerve ligation (SNL) within 3 hours [14]. However, the duration of action of (+)-naloxone was very short due to its brief half-life in the blood, although it showed analgesic effects in rats with long-established neuropathic pain at 8 weeks after surgery. Therefore, alternative TLR4 antagonists are required for long-term reversal of neuropathic pain with a single treatment rather than multiple treatments. Recently, screening of virtual libraries and phage display libraries may lead to the isolation of peptide TLR4 modulators capable of disrupting the TLR4/MD2 interaction or Toll/interleukin-1 receptor (TIR)/TIR interactions [15]. Here, we evaluated TLR4 antagonistic peptide 2 (TAP2) as a new analgesic agent for neuropathic pain, considering its safety and efficacy compared with LPS analogues and small compounds. In the present study, we examined whether TAP2, a peptide antagonist of TLR4, has an analgesic effect on SNL-induced neuropathic pain in rats. TLR4/MD2 complex-targeted TAP2 showed marked long-term attenuation of the mechanical allodynia in von Frey filament tests. Furthermore, the loss of neuropathic pain was attributed to the reduction in microglial activation and generation of pain inducers, such as proinflammatory mediators and reactive oxygen species (ROS), in the spinal dorsal horn of SNL-induced rats.

\section{MATERIALS AND METHODS}

\section{Animals}

Sprague-Dawley rats (male, 6-week-old, 150 200 g) were obtained from Daehan Bio Link (Chung-buk, Republic of Korea) and allowed to acclimatize to the new environment for 1 week before the experiments. All animals were housed (three rats per cage) in a controlled environment $\left(23 \pm 2^{\circ} \mathrm{C}, 50 \%\right.$ humidity) under 
a 12 h-light/dark cycle and given food and water ad libitum. In this study, 124 animals were used for the behavioral test (49), the analysis of gene expression analysis (28) and the immunohistochemical study (46). The animal experiments were approved by the Animal Care and Use Committee of Chungnam National University (approval number: CNUH-019-P0001) and conformed to the ethical guidelines of the National Institutes of Health and the International Association for the Study of Pain [16].

\section{Neuropathic pain model, pain behavioral test, and intra- thecal injection}

In this study, we used a rat model of neuropathic pain induced by SNL, which is a proved model of neuropathic pain in animals $[17,18]$. Briefly, under isoflurane anesthesia (2\% in oxygen; Hana Pharm, Kyung-gi, Republic of Korea), the animals were placed in the prone position, and the left side of the paraspinal muscles were spread wide apart via a 2 -cm skin incision. After elimination of the lumbar (L) 6 transverse process to reveal the L4 and L5 vertebrae, the L5 spinal nerve was carefully separated from L4 without injury and ligated three times using 3-0 silk thread (Ethicon, Diegem, Belgium). After ligation, the wound was sutured with silk thread, and the animals were allowed to recover on a warm pad. The sham group received the same surgical procedures as in the SNL group except that the L5 spinal nerve was not ligated.

Pain behavior was evaluated as described previously [16, 1921] using von Frey filaments (NC12775-99, Touch Test ${ }^{\circledR}$ Sensory Evaluators, 20-Piece Hand Kit; North Coast Medical \& Rehabilitation Products, Gilroy, CA). Briefly, rats were placed on a metal mesh floor covered with clear plastic boxes $(18 \times 8 \times 8 \mathrm{~cm})$ and left for 20 minutes to acclimatize to the new environment. Mechanical stimuli were applied with nine different von Frey filaments ranging from 0.6 to $15 \mathrm{~g}(0.6,1,1.4,2,4,6,8,10$, and $15 \mathrm{~g})$. Stimuli were applied with a von Frey filament in 3 4-second trials, each of which was repeated four times on each hind paw at intervals of approximately 5 minutes. The 2 -g filament stimulus was applied first. Then, mechanical threshold was determined with the up-down method [21]. If a positive response occurred, the next smaller von Frey filament was used. If a negative response occurred, the next larger filament was applied. The last filament applied was considered as a threshold of the test.

Intrathecal injection of compounds into the subarachnoid space of the spinal cord was performed as described previously [22]. Briefly, animals were placed in the prone position on an operating table under isoflurane anesthesia. Next, $20 \mu \mathrm{L}$ solution (PBS or TAP2 [AMALDCFRWGWRMWCSSG, Peptron, Daejeon, Republic of Korea], $25 \mathrm{nmol}$ ) was introduced intrathecally into the L5/L6 intervertebral space using a syringe ( $50 \mu \mathrm{L}$; Hamilton, Reno,
$\mathrm{NV}$ ) with a $26.5 \mathrm{G}$ needle. Further, fluorescein isothiocyanate (FITC)-conjugated TAP2 (FITC-TAP2, Peptron) was used to examine the microglial specificity and the duration of action in the spinal dorsal horn of SNL-induced rats. An obvious tail flick was observed when injection was successful.

\section{Quantitative real-time PCR analysis}

Total RNA was isolated from BV2 cells or the ipsilateral dorsal horn of the spinal cords (L4/L5 segment, $0.7 \mathrm{~cm}$ ) using the Hybrid-R kit (GeneAll, Seoul, Republic of Korea) [23] and then transcribed into cDNA using TOPscript RT DryMix (Enzynomics, Daejeon, Republic of Korea). qPCR was performed under the following conditions [24]: $95^{\circ} \mathrm{C}$ for 10 minutes, followed by 40 cycles of $95^{\circ} \mathrm{C}$ for 15 seconds and $60^{\circ} \mathrm{C}$ for 1 minute using the AriaMx Realtime PCR System (Agilent Technologies, Santa Clara, CA). Primer sequences (Cosmogenetech, Seoul, Republic of Korea) used in the qPCR were as follows: mouse GAPDH, forward: 5'ACC CAG AAG ACT GTG GAT GG -3', reverse: 5' - CAC ATT GGG GGT AGG AAC AC- -3'; mouse TNF- $\alpha$, forward: 5' - AGC AAA CCA CCA AGT GGA GGA-3', reverse: 5'- GCT GGC ACC ACT AGT TGG TTG T -3'; mouse IL-1 $\beta$, forward: 5'- TTG TGG CTG TGG AGA AGC TGT -3', reverse: 5'- AAC GTC ACA CAC CAG CAG GTT -3'; mouse IL-6, forward: 5' - TCC ATC CAG TTG CCT TCT TGG -3', reverse: 5' - CCA CGA TTT CCC AGA GAA CAT G -3'; mouse COX-2, forward: 5' - TGA GTA CCG CAA ACG CTT CT - 3', reverse: 5' - CTC CCC AAA GAT AGC ATC TGG - 3'; mouse iNOS, forward: 5' - GGC AAA CCC AAG GTC TAC GTT - 3', reverse: 5' - TCG CTC AAG TTC AGC TTG GT - 3'; rat GAPDH, forward: 5' - CTC ATG ACC ACA GTC CAT GC -3', reverse: 5'- TTC AGC TCT GGG ATG ACC TT -3'; rat TNF- $a$, forward: 5'- AGA TGT GGA ACT GGC AGA GG -3', reverse: 5' - CCC ATT TGG GAA CTT CTC CT -3'; rat IL$1 \beta$, forward: 5'- CAG CAG CAT CTC GAC AAG AG -3', reverse: 5'- CAT CAT CCC ACG AGT CAC AG -3'; rat IL-6, forward: 5'CCG GAG AGG AGA CTT CAC AG -3', reverse: 5'- ACA GTG CAT CAT CGC TGT TC -3'; rat COX-2 forward: 5' - CAG TAT CAG AAC CGC ATT GCC -3', reverse: 5'- GAG CAA GTC CGT GTT CAA GGA-3'; rat TLR4 forward: 5’- TGA GCA GTC GTG CTG GTA TC -3', reverse: 5'- CAG GGC TTT TCT GAG TCG TC -3. The mRNA levels of each target gene were normalized to that of GAPDH. The fold changes in mRNA levels were calculated using the $2^{-\Delta \Delta C t}$ method as described previously [25].

\section{Tissue preparation, immunohistochemistry and image analysis}

Rats were euthanized with sodium pentobarbital $(100 \mathrm{mg} / \mathrm{kg}$, i.p.), perfused with heparinized phosphate-buffered saline $(0.5 \%$ 
heparin in PBS, pH 7.4), followed by perfusion with ice-cold 4\% paraformaldehyde in PBS for 10 minutes using a peristaltic pump at a rate of $20 \mathrm{~mL} / \mathrm{min}$. The lumbar region (L4 L6) of the spinal cord was separated, bathed in the same fixative overnight at $4{ }^{\circ} \mathrm{C}$, and then immersed in a series of sucrose solutions in PBS (from $10 \%$ to $30 \%$ ) for cryoprotection [26]. After 2 days, the lumbar segments were cut into transverse sections at a thickness of $30 \mu \mathrm{m}$ using a cryostat (CM1950; Leica, Wetzlar, Germany) and kept at $-20^{\circ} \mathrm{C}$ in storage buffer (30\% glycerol, $30 \%$ ethylene glycol in PBS) [27].

For fluorescence staining, tissues were first blocked with blocking buffer (5\% normal serum, $0.3 \%$ Triton X-100 in PBS) for 1 hour at room temperature. The spinal tissues were then incubated with a mixture of primary antibodies (iNOS, 1:100 [BD-610329; BD Transduction Laboratories, Franklin Lakes, NJ]; Iba1, 1:400 [019-19741; Wako, Osaka, Japan]); diluted in blocking buffer overnight at $44^{\circ} \mathrm{C}$ followed by a mixture of corresponding secondary antibodies conjugated with either FITC or $\mathrm{Cy}^{3}$ (1:400; Jackson ImmunoResearch, West Grove, PA) diluted in the same blocking buffer [28]. The sections were counterstained with DAPI (5 $\mu \mathrm{g} /$ $\mathrm{mL}$ in PBS; Thermo Fisher Scientific, Waltham, MA) for 5 minutes and then mounted on glass slides with mounting medium (Biomeda, Foster City, CA). Images were obtained using a laser scanning microscope (TCS SP8; Leica) and used for additional data analysis.

For diaminobenzidine (DAB) staining, the spinal tissues were first exposed to $0.3 \% \mathrm{H}_{2} \mathrm{O}_{2}$ in PBS for 10 minutes to block endogenous peroxidase activity and incubated with the same blocking solution for 1 hour at room temperature. The tissues were then treated with the primary antibody (Iba-1, 1:400; Wako) overnight at $4^{\circ} \mathrm{C}$ followed by a biotinylated secondary antibody and the streptavidin peroxidase complex (Vector Laboratories, Inc., Burlingame, $\mathrm{CA})$. The specimens were visualized with $\mathrm{DAB}$-peroxidase substrate solution $\left(0.05 \% \mathrm{DAB} / 0.015 \% \mathrm{H}_{2} \mathrm{O}_{2}\right.$ in PBS) and mounted on glass slides using Polymount (Polysciences, Warrington, PA). Images were obtained by bright field microscopy (ECLIPSE E600 POL; Nikon, Tokyo, Japan).

The intensity of Ibal-immunoreactive (IR), FITC- positive, and DHE-positive cells in the spinal dorsal horn was measured using Image J software (NIH, Bethesda, MD) as described previously [16, 29]. In brief, images ( 3 sections per animal, $n=3 \sim 5$ animals) were first converted to gray ones. After background subtraction and shading correction, the intensity of rectangular area $(650 \mu \mathrm{m} \times 150$ $\mu \mathrm{m})$ in the layer I-IV of spinal dorsal horn was measured. In addition, the number of FITC-TAP2/Iba1-IR, iNOS-IR, and iNOS/ Ibal-IR cells was counted in the layer I-IV of dorsal horn of the spinal cord.

\section{Reactive oxygen species (ROS) detection assay}

Superoxide anion levels in the spinal cord were examined with dihydroethidium (DHE, Thermo Fisher Scientific), as described previously [30]. Briefly, spinal sections were incubated with DHE $(1 \mu \mathrm{M})$ solution for $5 \mathrm{~min}$ at room temperature and mounted on glass slides. The signals were grabbed with a confocal microscope (Leica Biosystems, Germany), and the fluorescent intensity was quantified with the Image J program.

\section{Monoiodoacetate (MIA)-induced knee osteoarthritic (OA) pain model, intrathecal injection, and behavioral test}

A rat model of OA pain was established by intraarticular injection of MIA (Sigma, St. Louis, MO) as described previously [31]. Briefly, under isoflurane anesthesia (2\% in oxygen mixture; Hana Pharm, Kyung-gi, Republic of Korea), PBS ( $25 \mu \mathrm{L})$ or MIA ( 2 mg in $25 \mu \mathrm{L}$ saline) was added to the intraarticular space of the knee joint of the left hind leg through the left patellar tendon using a Hamilton syringe with a $26.5 \mathrm{G}$ needle. Once OA pain was established on day 7 post-MIA injection, PBS or TAP2 $(25 \mathrm{nmol})$ was delivered intrathecally into the rats with the same procedure of SNL model above. The animals then underwent behavioral tests with von Frey filaments on the indicated days to validate the analgesic effect of TAP2 on OA pain. Furthermore, to examine the suppressive effect of TAP2 on spinal microglia in an OA pain model, on day 10 after TAP2 injection, L5 spinal tissues were immunostained with antiIbal antibodies.

\section{Statistical analysis}

The data are expressed as the mean \pm standard error of the mean (SEM). The significance of differences between groups was compared by one-way or two-way analysis of variance (ANOVA), followed by the appropriate multiple comparison test. Two-group analyses were performed using the two-tailed unpaired Student's $t$ test. Statistical analyses were performed using GraphPad Prism 6 (GraphPad Software Inc., La Jolla, CA). In all analyses, $\mathrm{p}<0.05$ was taken to indicate statistical significance.

\section{RESULTS}

\section{TLR4 expression is increased in rats with SNL-induced neuropathic pain}

In mouse models of neuropathic pain with spinal nerve transection and chronic construction injury, neuropathic pain is mediated by TLR 4 and its downstream molecules $[10,11]$. However, there have been no reports regarding whether neuropathic pain is also mediated by TLR4 in rats. Therefore, we first examined the expression of TLR4 in rats with neuropathic pain. 
To verify the mRNA level of TLR4 in rats with SNL-induced neuropathic pain, we first performed L5 SNL ligation in rats insensitive to von Frey filaments (>10 g, baseline) and confirmed the incidence and continuation of neuropathic pain by von Frey filament test on days 3, 7, and 14 (Fig. 1A). The level of TLR4 transcription in the L4-L5 segments of the ipsilateral spinal dorsal horn was then investigated by qPCR using primers specific for TLR4. The results indicate that the TLR4 mRNA level was upregulated by threefold on day 3 post-ligation in SNL-induced rats and subsequently downregulated (Fig. 1B).

These observations suggest that neuropathic pain may involve TLR4 due to the increased TLR4 expression in the rat model of neuropathic pain with SNL.

\section{TAP2 suppresses the gene expression of proinflammatory} mediators in LPS-stimulated BV2 cells

Prior to the administration of TAP2 in rats with SNL-induced neuropathic pain, we investigated the anti-inflammatory effects of TAP2 on microglial cells because TLR4 is upregulated on the surface of spinal microglia in animals with neuropathic pain [6]. BV2 cells were pre-treated with TAP2 $(10 \mu \mathrm{M})$ for $30 \mathrm{~min}$ and further incubated with LPS (100 ng/ml) for $3 \mathrm{~h}$. The mRNA levels of the proinflammatory mediators, such as TNF- $\alpha$, IL- $1 \beta$, IL-6, COX-2, and iNOS, were markedly decreased by $54-83 \%$ in the LPS+TAP2 -treated group compared with the LPS-treated group in qPCR analysis (Fig. 2).
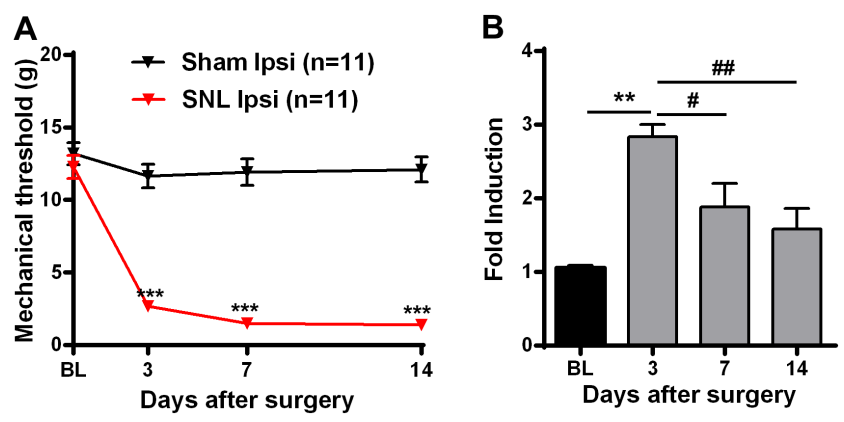

Fig. 1. TLR4 expression was significantly increased in the ipsilateral dorsal horn of rats with SNL-induced neuropathic pain. (A) To establish a rat model of neuropathic pain, rats sensitive to von Frey filaments (>10 g filaments) were subjected to lumbar 5 (L5) SNL. Then, the von Frey filament test was performed on days 3,7, and 14 post-ligations. Data are presented as means \pm SEM (one-way analysis of variance (ANOVA) with Dunnett's post hoc test, ${ }^{* * *} \mathrm{p}<0.001$ vs. BL of SNL Ipsi; $\mathrm{n}=11$ per group). (B) On days 3,7 , and 14 after surgery, the L4-6 segments of the ipsilateral dorsal horn were processed and used for isolation of total RNA. After cDNA synthesis, the TLR4 mRNA level was determined by qPCR analysis. Sham operation was performed as a control. Data are presented as means \pm SEM (oneway ANOVA with Tukey's post hoc test, ${ }^{* *} \mathrm{p}<0.01 \mathrm{vs}$. BL, \#\# $<0.01$ and $\# \mathrm{p}<0.05 ; n=4-5$ per group). BL, baseline; Ipsi, Ipsilateral.
These findings indicate that TAP2 has an anti-inflammatory effect on microglia in vitro and is a candidate for the treatment of neuropathic pain via suppression of microglial activation.

\section{TAP2 effectively alleviates mechanical allodynia induced by SNL in rats by decreasing microglial activation}

Before validating the analgesic effect of TAP2 on neuropathic pain, we investigated whether TAP2 preferentially targets spinal microglia in rats with neuropathic pain because TLR4 expression is markedly upregulated in microglia after surgery [11]. To track the localization of TAP2 in the spinal cord, FITC was conjugated to the N-terminus of TAP2 (FITC-TAP2) and administered in-

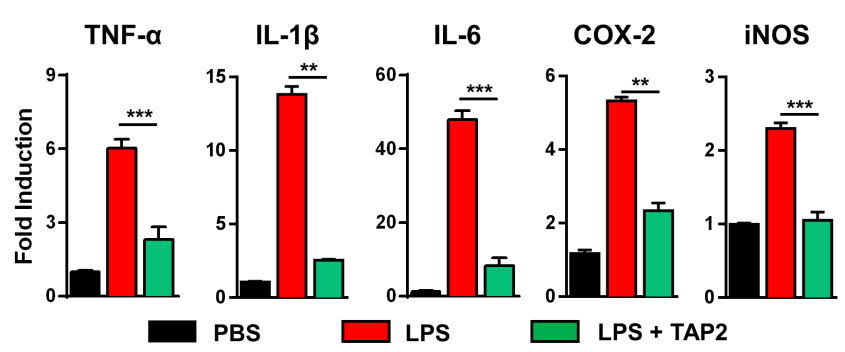

Fig. 2. TAP2 suppressed the gene expression of proinflammatory mediators in LPS-stimulated BV2 cells. After pretreatment with TAP2 $(10 \mu \mathrm{M})$ for 30 minutes, BV2 cells were further incubated with LPS $(100 \mathrm{ng} / \mathrm{mL})$ for 3 hours. The mRNA levels of proinflammatory mediators, including TNF- $\alpha$, IL- $1 \beta$, IL- 6 , COX-2, and iNOS, were measured by qPCR. Data are presented as means \pm SEM (one-way ANOVA with Tukey's post hoc test, ${ }^{* * *} \mathrm{p}<0.001$ and ${ }^{* *} \mathrm{p}<0.01$ vs. LPS; $\mathrm{n}=3$ per group). LPS, lipopolysaccharide.
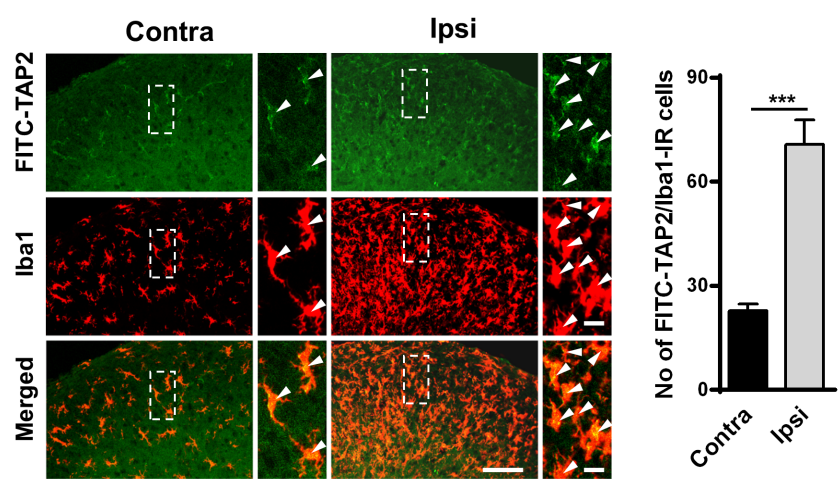

Fig. 3. FITC-conjugated TAP2 preferentially targeted spinal microglia in rats with SNL-induced neuropathic pain. On day 3 after surgery, FITCconjugated TAP2 (FITC-TAP2) was administered intrathecally to SNLinduced rats. After 4 days, L5 spinal sections were prepared and incubated with an antibody against the microglia marker Ibal. Arrowheads indicate the microglial cells targeted by FITC-conjugated TAP2 in the spinal dorsal horn. Scale bar=500 $\mu \mathrm{m}$ (left), $200 \mu \mathrm{m}$ (right). The numbers of Iba1/ FITC-TAP2-positive cells were quantified in the contralateral (Contra) and ipsilateral (Ipsi) spinal dorsal horn. Data are presented as means \pm SEM (unpaired Student's $t$ test, ${ }^{* * *} \mathrm{p}<0.001$ vs. Contra; $\mathrm{n}=3$ per group). 
trathecally to rats with SNL-induced neuropathic pain on day 3 after surgery. Immunofluorescent staining indicated that TAP2 was exclusively localized in Iba1-positive microglia of the spinal cord on day 4 post-injection (Fig. 3). Interestingly, the number of TAP2/Ibal-positive cells in the spinal dorsal horn was markedly increased by three-fold on the ipsilateral side compared with the contralateral side (Fig. 3).

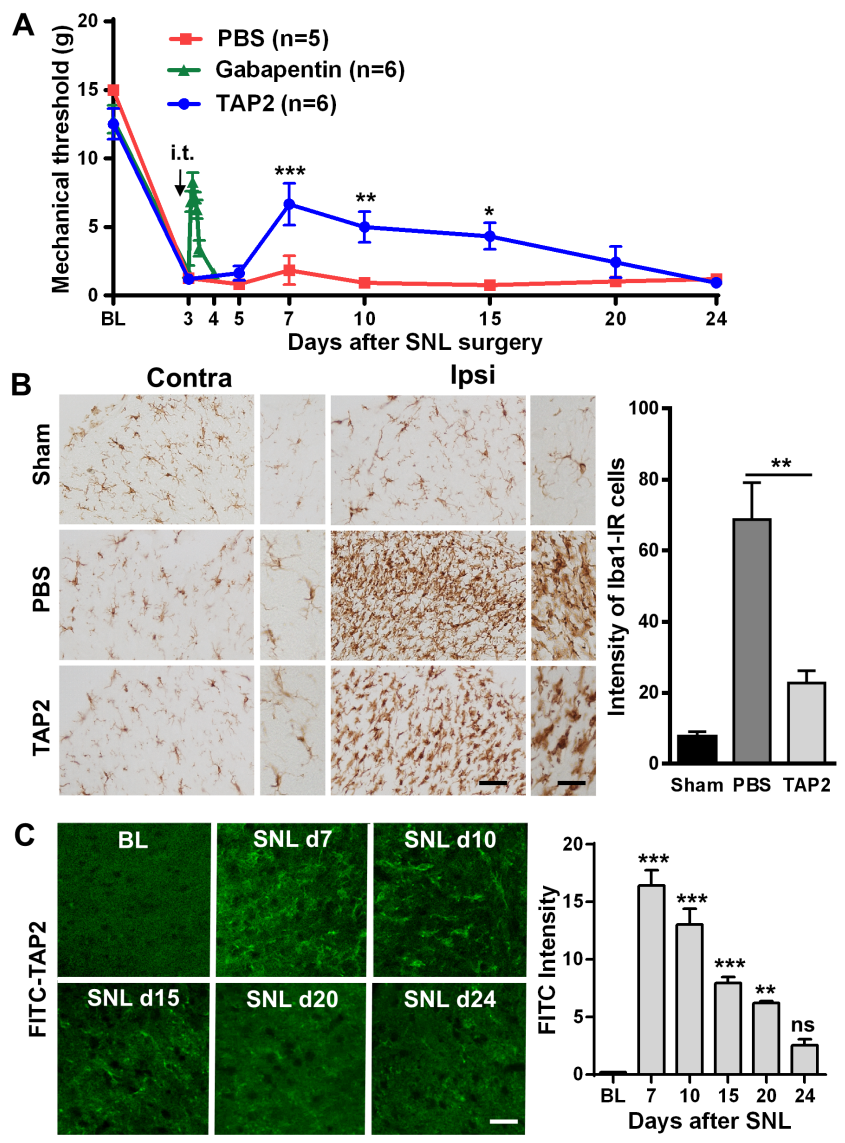

Fig. 4. TAP2 efficiently alleviated mechanical allodynia induced by SNL in rats by decreasing microglial activation. (A) On day 3 after surgery, sensitive rats ( $<4 \mathrm{~g}$ filaments) were randomly divided into two groups, followed by intrathecal administration of PBS or TAP2 $(25 \mathrm{nmol}$ in $20 \mu \mathrm{L}$ PBS) directly into the L5/L6 intervertebral space. von Frey filament tests were then performed on days 5,7,10,15,20, and 24 after surgery. Data are presented as means \pm SEM (two-way ANOVA with Tukey's post hoc test, ${ }^{* * *} \mathrm{p}<0.001,{ }^{* *} \mathrm{p}<0.01$, and ${ }^{*} \mathrm{p}<0.05$ vs. PBS; $\mathrm{n}=5-6$ per group). Gabapentin was used as a control. (B) On day 4 post-injection of PBS or TAP2, L5 spinal tissues were prepared and incubated with anti-Ibal antibodies. Scale bar $=200 \mu \mathrm{m}$ (left), $50 \mu \mathrm{m}$ (right). The intensity of Ibal immunoreactivity in the spinal dorsal horn was measured. Sham operation was performed as a control. Data are presented as means \pm SEM (one-way ANOVA with Tukey's post hoc test, ${ }^{* *} \mathrm{p}<0.01$ vs. $\mathrm{PBS} ; \mathrm{n}=3$ per group). (C) On days 7,10 , 15, 20, and 24 after SNL surgery (on days 4, 7, 12, 17, and 21 after FITCTAP2 delivery), the green intensity in the ipsilateral side of L5 spinal dorsal horns was measured. Scale bar $=200 \mu \mathrm{m}$ (left). Data are presented as means \pm SEM (one-way ANOVA with Dunnett's post hoc test, ${ }^{* * *} \mathrm{p}<0.001$ and ${ }^{* *} \mathrm{p}<0.01$ vs. $\mathrm{BL} ; \mathrm{n}=3$ per group). BS, baseline; $\mathrm{ns}$, not significant.
Next, to determine whether TAP2 mitigates SNL-induced pain in rats, on day 3 after surgery, rats sensitive to von Frey filaments $(<4$ g) were randomly divided into two groups and treated by intrathecal injection of PBS $(20 \mu \mathrm{L})$ or TAP2 $(25 \mathrm{nmol}$ in $20 \mu \mathrm{L}$ PBS $)$ (Fig. 4A). The von Frey filament test performed on days 5, 7, 10, 15, 20, and 24 after ligation indicated that the analgesic effect of TAP2 on neuropathic pain was significantly maintained for approximately 2 weeks compared with the control group. Interestingly, the analgesic effect of TAP2 lasted much longer than that of gabapentin (5 $\mathrm{mg}$ ), which is used clinically for the treatment of neuropathic pain (Fig. 4A).

Microglial activation involved in neuropathic pain is mediated by TLR4 and its downstream signaling pathways $[11,32]$. Therefore, we examined the reduction in microglial activation induced by TAP2 in SNL-induced rats because TAP2 targets TLR4/MD2 complex expressed on the surface of microglia. On day 4 postinjection of PBS or TAP2, L5 spinal sections were immunostained with anti-Ibal antibodies. The intensity of Ibal-immunoreactive cells was decreased by $67 \%$ in the TAP2-treated group compared with the PBS-treated controls (Fig. 4B).

Furthermore, we investigated the relationship between the reduction of pain and the duration of TAP2 in the spinal cord of SNL-induced rats. Thus, on days 4, 7, 12, 17, and 21 following administration of FITC-TAP2 to SNL-induced rats, the intensity of green fluorescence was measured on the targeted cells of ipsilateral spinal dorsal horn. Interestingly, mechanical allodynia decreased in reverse proportion to the intensity of FITC-TAP2 in the spinal dorsal horn of rats induced by SNL surgery (Fig. 4C).

Taken together, these observations suggest that TAP2 substantially targets microglia in the spinal cord and achieves long-term mitigation of SNL-induced pain in rats by reducing microglial activation.

\section{TAP2 relieves neuropathic pain by decreasing the levels of proinflammatory mediators and ROS in the spinal dorsal horn}

To understand the mechanism underlying the reduction in neuropathic pain by TAP2, we examined the expression levels of the proinflammatory mediators, such as TNF- $\alpha$, IL- $1 \beta$, IL- 6 , COX-2, and iNOS, because the expression levels of these genes are noticeably upregulated in the spinal dorsal horn of animals with neuropathic pain [3]. On day 4 post-injection, the mRNA levels of TNF- $\alpha$, IL- $1 \beta$, and IL- 6 in the ipsilateral spinal dorsal horn were significantly decreased by $52-71 \%$ in the TAP2-treated group compared with the PBS-treated controls (Fig. 5A). Furthermore, the numbers of iNOS- and iNOS/Ibal-immunopositive cells in the ipsilateral spinal dorsal horn were also markedly decreased by 
$39 \%$ and $46 \%$, respectively, in the TAP2-treated group compared with the controls (Fig. 5B).

As SNL-induced microglial activation enhances the generation of ROS [33], we also examined whether TAP2 inhibits ROS production caused by the blockade of TLR4/MD2 complex and subsequent decrease in microglial activation. When L5 spinal sections were incubated with a ROS marker, dihydroethidium, the level of ROS in the dorsal horn of the spinal cord was significantly decreased by $55 \%$ in the TAP2-treated group compared with the PBS-treated controls (Fig. 6).

Together, our findings suggest that the alleviation of neuropathic pain induced by TAP2 may be attributed to decreased levels of proinflammatory molecules and ROS in the spinal dorsal horn.
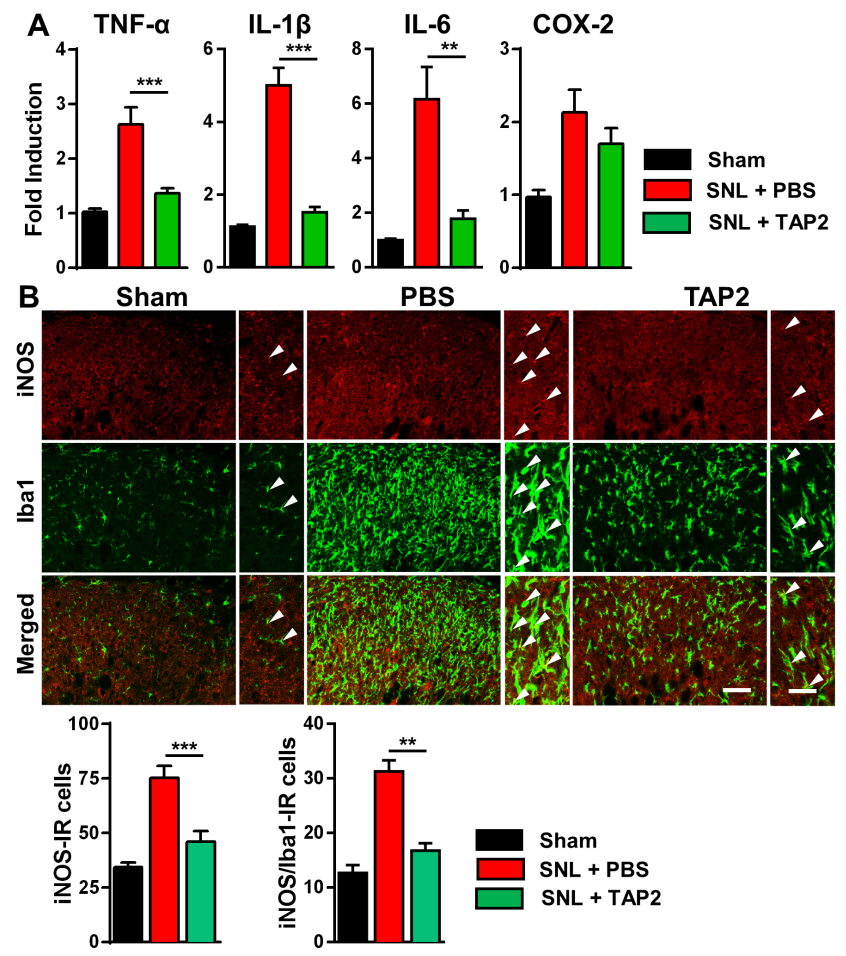

Fig. 5. Attenuation of neuropathic pain by TAP2 was attributed to the loss of proinflammatory mediators in the spinal dorsal horn. (A) On day 4 after introduction of PBS or TAP2 $(25 \mathrm{nmol})$ into SNL-induced rats, total RNA was isolated from the L4/5 segments $(0.7 \mathrm{~cm})$ of the ipsilateral dorsal horn in the spinal cord. The mRNA levels of proinflammatory mediators, including TNF- $\alpha$, IL- $1 \beta$, IL- 6 , and COX-2, were determined by qPCR. Sham operation was performed as a control. Data are expressed as means \pm SEM (one-way ANOVA with Tukey's post hoc test, ${ }^{* * *} \mathrm{p}<0.001$ and ${ }^{* *} \mathrm{p}<0.01$, vs. SNL+PBS; $\mathrm{n}=6$ per group). (B) The $\mathrm{L} 5$ spinal tissues were double-immunostained with anti-Ibal and anti-iNOS antibodies. The numbers of iNOS- and iNOS/Ibal-immunopositive cells in the layer I IV of ipsilateral spinal dorsal horn were presented. Arrowheads indicate iNOS/Ibal-positive cells. Scale bar=200 $\mu \mathrm{m}$ (left), $50 \mu \mathrm{m}$ (right). Data are presented as means \pm SEM (one-way ANOVA with Tukey's post hoc test, ${ }^{* * *} \mathrm{p}<0.001$ and ${ }^{* *} \mathrm{p}<0.01$ vs. $\mathrm{SNL}+\mathrm{PBS} ; \mathrm{n}=4-5$ per group). IR, immunoreactive.
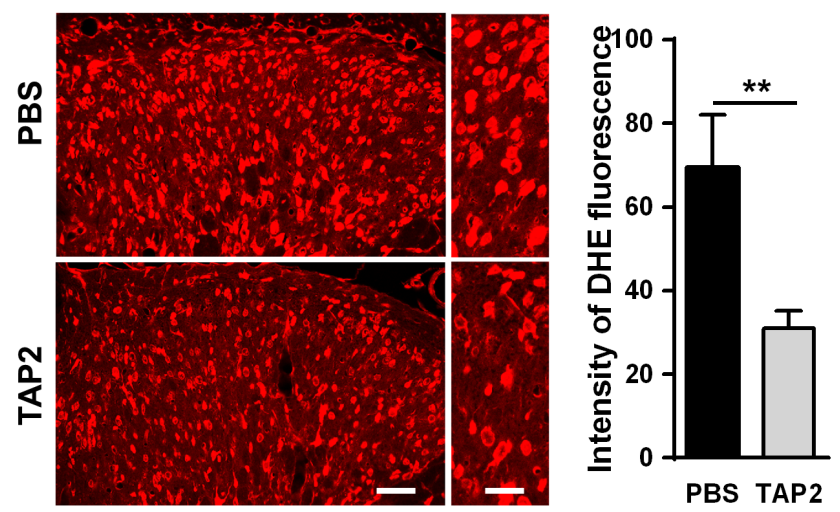

Fig. 6. TAP2 also decreases ROS level in the spinal dorsal horn in SNLinduced rats. On day 4 post-injection of PBS and TAP2, L5 spinal tissues were incubated with DHE solution $(1 \mu \mathrm{M})$ to visualize ROS. Scale bars $=500 \mu \mathrm{m}$ (left), $200 \mu \mathrm{m}$ (right). The ROS level in spinal dorsal horn was denoted as the intensity of DHE fluorescence. Data are expressed as the mean \pm SEM (unpaired Student's $t$-test, ${ }^{* *} \mathrm{p}<0.01$ vs. PBS; $\mathrm{n}=3-5$ per group). DHE, dihydroethidium.
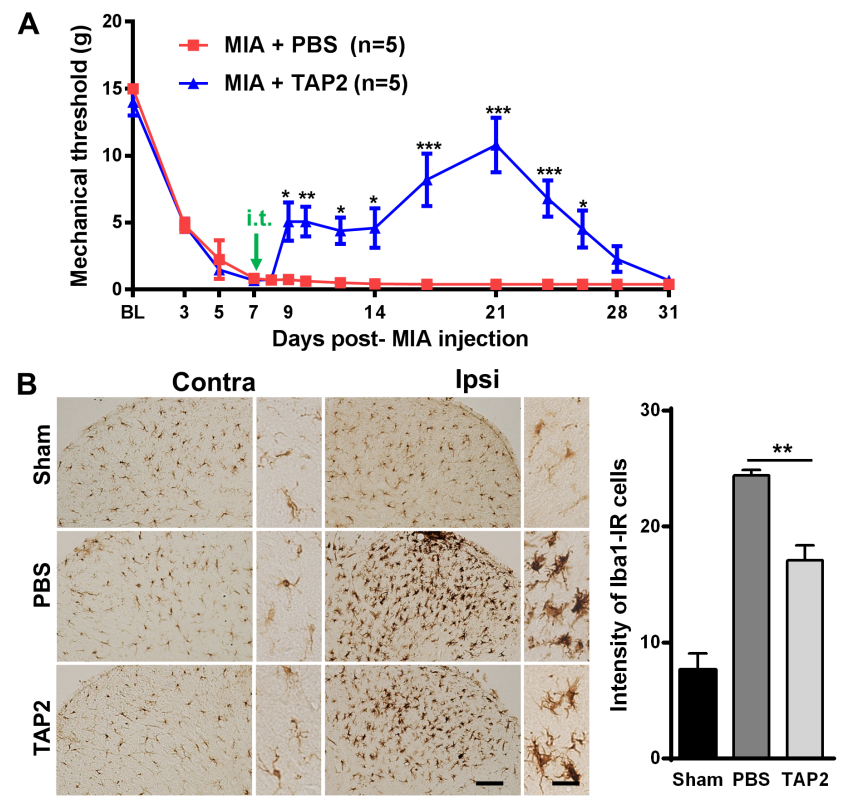

Fig. 7. MIA-induced osteoarthritic pain was markedly relieved by intrathecal injection of TAP2 by inhibiting microglia activation. (A) On day 7 post-injection of MIA ( $2 \mathrm{mg}$ in $25 \mu \mathrm{L}$ PBS) into the knee joint of the left hind leg, rats were randomly divided into two groups and intrathecally administered PBS or TAP2 (25 nmol in $20 \mu \mathrm{L}$ PBS). The mechanical threshold was then examined from days 1 to 24 post-injection. Data are expressed as means \pm SEM (two-way ANOVA with Bonferroni post hoc test, ${ }^{* *} \mathrm{p}<0.001,{ }^{* *} \mathrm{p}<0.01$, and ${ }^{*} \mathrm{p}<0.05$ vs. MIA $+\mathrm{PBS} ; \mathrm{n}=5$ per group). (B) On day 10 after TAP2 injection, L5 spinal tissues were immunostained with anti-Ibal antibodies. Scale bar $=200 \mu \mathrm{m}$ (left), $50 \mu \mathrm{m}$ (right). The intensity of Ibal-immunoreacitve cells in the spinal dorsal horn was measured and presented. Sham operation was performed as a control. Data are presented as means \pm SEM (one-way ANOVA with Tukey's post hoc test, ${ }^{* *} \mathrm{p}<0.01$ vs. $\mathrm{PBS} ; \mathrm{n}=3$ per group). 
TAP2 efficiently also mitigates osteoarthritic pain behavior induced by MIA in rats with the reduction of microglia activation

Finally, to evaluate the therapeutic effect of TAP2 in a physiologically relevant disease model of neuropathic pain, we used an OA model because a subpopulation ( 30\%) of OA patients show neuropathic-like pain $[34,35]$. To establish the rat model of OA pain, MIA (2 mg in $25 \mu \mathrm{L}$ PBS) was administered intra-articularly into the knee of the hind leg, which has been shown to induce OA via gradual death of chondrocytes in the cartilage of the knee joint, mediated by inhibition of ATP production in glycolysis [31]. On day 7 post-MIA injection, PBS or TAP2 (25 nmol in $25 \mu \mathrm{L}$ PBS) was administered intrathecally to rats with OA pain. The mechanical thresholds were then determined using von Frey filaments on days $1,2,3,5,7,10,14,17,19,21$, and 24 post-injection. Similar to its effect on SNL-induced pain, TAP2 significantly improved MIAinduced pain behavior for 19 days compared with PBS control treatment (Fig. 7A).

Further, we investigated whether TAP2 could reudce OA pain by inhibiting microglia activation. When L5 spinal tissues were stained with anti-Ibal antidodies on day 10 post-TAP2 injection, Iba1-immunoreactivity was decreased by $30 \%$ in the TAP2-treated group compared with the PBS-treated controls (Fig. 7B).

These observations indicate that TAP2 causes long-term attenuation of OA pain induced by MIA in rats by prohibiting microglia activation. Therefore, TAP2 is a potential candidate for the treatment of patients with neuropathic pain, including those with OA.

\section{DISCUSSION}

In the present study, we examined whether an antagonistic peptide fragment of TLR4 is a more effective analgesic for the treatment of neuropathic pain compared with other drugs, because TLR4-mediated neuroinflammation contributes to the induction and conservation of neuropathic pain [6]. TAP2 administered intrathecally to the L5/L6 intervertebral space of rats with SNLinduced pain on day 3 after surgery markedly reduced pain behavior for approximately 2 weeks in von Frey filament tests, with the loss of microglial activation and subsequent downregulation of ROS and proinflammatory mediators, including TNF- $\alpha$, IL- $1 \beta$, IL6, COX-2, and iNOS, in the ipsilateral spinal dorsal horn.

In the innate immune system, TLR4 expressed on the surface of immune cells, including microglia, macrophages, and dendritic cells, recognizes the LPS on gram-negative bacteria and the envelope proteins of the respiratory syncytial virus [36] and ultimately provides host protection against foreign microorganisms [4]. Interestingly, TLR4 can also sense endogenous cellular components derived from injured tissues in vivo. In mouse models of neuropathic pain induced by CCI and SNL $[10,11]$, TLR4 expression in microglia was notably increased in the dorsal horn of the spinal cord, in response to substances released from nerve terminals of damaged sensory neurons $[7,8]$. In this study, TLR4 was also upregulated in the spinal dorsal horn of rats with SNL. Therefore, we used a model of neuropathic pain in rats induced with SNL to test the analgesic effect of TLR4 antagonists.

Initially, TLR4 antagonists, such as Eritoran and FP7, were used to treat infectious diseases, such as sepsis, lethal influenza infection, and inflammatory bowel disease [13]. Later, other TLR4 blockers were also validated as anesthetics for chronic pain, which is actually mediated by neuroinflammation via TLR4 in the spinal cord. However, they had a short duration (3 hours) of action on neuropathic pain in rats with CCI and SNL due to its instability in the blood [14]. Therefore, as alternative to previous LPS derivatives and small compounds, we searched for agents with longer halflives in vivo after a single treatment.

Small peptide antagonists of TLR4 may be better candidates for blocking TLR4-intermediated chronic pain because they are more stable than synthetic compounds. As a novel therapeutic agent to satisfy these requirements for treating neuropathic pain, we investigated whether TAP2 isolated from a phage display library [15] can alleviate chronic pain, as TAP2 showed a strong antiinflammatory effect on LPS-elicited RAW264.7 cells by suppressing the activation of NF- $\kappa \mathrm{B}$ and MAPKs in TLR4-mediated signal transduction [15]. Prior to TAP2 treatment of animals with neuropathic pain in vivo, we verified that TAP2 markedly inhibited the mRNA levles of the proinflammatory mediators, including IL6, COX-2, and iNOS, in LPS-activated BV2 cells. Interestingly, to track the localization of TAP2 in the spinal cord of rats with neuropathic pain, when FITC-TAP2 was intrathecally delivered into SNL-induced rats, they co-localized with spinal microglia, preferentially activated microglia of ipsilateral dorsal horn. Furthermore, after a single intrathecal administration of TAP2 to SNL-induced rats, pain behavior in the von Frey filament test was significantly improved for about 2 weeks. The reduction in chronic pain was attributed to the decreased microglial activation and, subsequently, levels of neuropathic pain-related factors, such as proinflammatory mediators and ROS. To know the relation between the loss of mechanical threshold and the persistent of TAP2 in the spinal dorsal horn, we administered FITC-TAP2 on day3 post-surgery to SNL-induced rats and then examined the intensity of FITC on L5 spinal tissues for 20 days. Curiously, mechanical threshold was reversely proportional to the intensity of FITC-TAP2 in the spinal dorsal horn of rats with SNL-induced pain. Finally, we found that, in a clinical model of OA pain induced by MIA [34, 35], TAP2 also 
relieved mechanical allodynia for 19 days by preventing microglia activation with the similar pattern of SNL model.

Although a single treatment with TAP2 had a long-term analgesic effect in animal models of neuropathic pain elicited by SNL and MIA, it is still inconvenient. Although intrathecal injection mitigated chronic pain for 2 weeks, it is necessary to extend the duration of pain alleviation because patients are unlikely to accept the injection procedure. One solution is the use of nanoparticles enclosed in nanomaterials, such as poly (D,L-lactic-co-glycolic acid) or liposomes, to increase the release time of TAP2 in the cerebrospinal fluid. Further, TAP2 cannot easily penetrate the blood-brain barrier. This can be enhanced by the addition and modification of amino acids at the $\mathrm{N}$ - and C-termini of TAP2 [37]. In addition, there were issues for the efficacy and safety of TLR4 antagonists, such as Eritoran and Resatorvid, in clinical trials. These TLR4 candidates were tested with high dose in the patients with sepsis, an extreme inflammatory condition. However, TAP2 may have high efficacy and less toxicity in several ways: 1) TAP2 was tested with low dose in a model of neuropathic pain with mild inflammation. 2) TAP2 was originated from peptides, which is the natural substance. 3) TAP2 is locally (intrathecally) treated to minimize the its side effects.

In summary, the findings from the present study indicate that a small antagonistic peptide of TLR4, TAP2, markedly mitigated chronic pain induced by SNL in rats via the loss of microglial activation, followed by decreased production of pronociceptive factors, such as proinflammatory mediators and ROS, due to inhibition of TLR4/MD-2 complex-mediated signal transduction. Therefore, it represents a promising candidate as an analgesic for the treatment of neuropathic pain.

\section{ACKNOWLEDGEMENTS}

This research was supported by the Brain Research Program through the National Research Foundation of Korea (NRF) funded by the Ministry of Science, ICT \& Future Planning (NRF-2016M3C7A1905074, 2016R1A2B4009409, 2017R1D1A1B03028839, 2017R1A2B4005905, 2017R1A1A1A05001310).

\section{REFERENCES}

1. Anand P, Shenoy R, Palmer JE, Baines AJ, Lai RY, Robertson J, Bird N, Ostenfeld T, Chizh BA (2011) Clinical trial of the p38 MAP kinase inhibitor dilmapimod in neuropathic pain following nerve injury. Eur J Pain 15:1040-1048.

2. Austin PJ, Moalem-Taylor G (2010) The neuro-immune balance in neuropathic pain: involvement of inflammatory immune cells, immune-like glial cells and cytokines. J Neuroimmunol 229:26-50.

3. Marchand F, Perretti M, McMahon SB (2005) Role of the immune system in chronic pain. Nat Rev Neurosci 6:521-532.

4. Kumar H, Kawai T, Akira S (2009) Pathogen recognition in the innate immune response. Biochem J 420:1-16.

5. Schaefer L (2014) Complexity of danger: the diverse nature of damage-associated molecular patterns. J Biol Chem 289:35237-35245.

6. Raghavendra V, Tanga F, DeLeo JA (2003) Inhibition of microglial activation attenuates the development but not existing hypersensitivity in a rat model of neuropathy. J Pharmacol Exp Ther 306:624-630.

7. Nicotra L, Loram LC, Watkins LR, Hutchinson MR (2012) Toll-like receptors in chronic pain. Exp Neurol 234:316-329.

8. Lacagnina MJ, Watkins LR, Grace PM (2018) Toll-like receptors and their role in persistent pain. Pharmacol Ther 184:145-158.

9. Maqboul A, Elsadek B (2018) Expression profiles of TRPV1, TRPV4, TLR4 and ERK1/2 in the dorsal root ganglionic neurons of a cancer-induced neuropathy rat model. PeerJ 6:e4622.

10. Piao Y, Gwon DH, Kang DW, Hwang TW, Shin N, Kwon HH, Shin HJ, Yin Y, Kim JJ, Hong J, Kim HW, Kim Y, Kim SR, Oh SH, Kim DW (2018) TLR4-mediated autophagic impairment contributes to neuropathic pain in chronic constriction injury mice. Mol Brain 11:11.

11. Tanga FY, Nutile-McMenemy N, DeLeo JA (2005) The CNS role of Toll-like receptor 4 in innate neuroimmunity and painful neuropathy. Proc Natl Acad Sci U S A 102:5856-5861.

12. Kuzmich NN, Sivak KV, Chubarev VN, Porozov YB, Savateeva-Lyubimova TN, Peri F (2017) TLR4 signaling pathway modulators as potential therapeutics in inflammation and sepsis. Vaccines (Basel) 5:E34.

13. Neal MD, Jia H, Eyer B, Good M, Guerriero CJ, Sodhi CP, Afrazi A, Prindle T Jr, Ma C, Branca M, Ozolek J, Brodsky JL, Wipf P, Hackam DJ (2013) Discovery and validation of a new class of small molecule Toll-like receptor 4 (TLR4) inhibitors. PLoS One 8:e65779.

14. Lewis SS, Loram LC, Hutchinson MR, Li CM, Zhang Y, Maier SF, Huang Y, Rice KC, Watkins LR (2012) (+)-naloxone, an opioid-inactive toll-like receptor 4 signaling inhibitor, reverses multiple models of chronic neuropathic pain in rats. J Pain 13:498-506.

15. Park S, Shin HJ, Shah M, Cho HY, Anwar MA, Achek A, Kwon HK, Lee B, Yoo TH, Choi S (2017) TLR4/MD2 specific peptides stalled in vivo LPS-induced immune exacerbation. Biomaterials 126:49-60. 
16. Zhang E, Yi MH, Ko Y, Kim HW, Seo JH, Lee YH, Lee W, Kim DW (2013) Expression of LC3 and Beclin 1 in the spinal dorsal horn following spinal nerve ligation-induced neuropathic pain. Brain Res 1519:31-39.

17. Kim SH, Chung JM (1992) An experimental model for peripheral neuropathy produced by segmental spinal nerve ligation in the rat. Pain 50:355-363.

18. Ma Z, Han Q, Wang X, Ai Z, Zheng Y (2016) Galectin-3 inhibition is associated with neuropathic pain attenuation after peripheral nerve injury. PLoS One 11:e0148792.

19. Chung JM, Kim HK, Chung K (2004) Segmental spinal nerve ligation model of neuropathic pain. Methods Mol Med 99:3545.

20. Pitcher GM, Ritchie J, Henry JL (1999) Paw withdrawal threshold in the von Frey hair test is influenced by the surface on which the rat stands. J Neurosci Methods 87:185-193.

21. Chaplan SR, Bach FW, Pogrel JW, Chung JM, Yaksh TL (1994) Quantitative assessment of tactile allodynia in the rat paw. J Neurosci Methods 53:55-63.

22. Hylden JL, Wilcox GL (1980) Intrathecal morphine in mice: a new technique. Eur J Pharmacol 67:313-316.

23. Zhang E, Lee S, Yi MH, Nan Y, Xu Y, Shin N, Ko Y, Lee YH, Lee W, Kim DW (2017) Expression of granulocyte colonystimulating factor 3 receptor in the spinal dorsal horn following spinal nerve ligation-induced neuropathic pain. Mol Med Rep 16:2009-2015.

24. Cho IH, Hong J, Suh EC, Kim JH, Lee H, Lee JE, Lee S, Kim CH, Kim DW, Jo EK, Lee KE, Karin M, Lee SJ (2008) Role of microglial IKKbeta in kainic acid-induced hippocampal neuronal cell death. Brain 131:3019-3033.

25. Livak KJ, Schmittgen TD (2001) Analysis of relative gene expression data using real-time quantitative PCR and the 2(-Delta Delta C(T)) Method. Methods 25:402-408.

26. Ito S, Suto T, Saito S, Obata H (2018) Repeated administration of duloxetine suppresses neuropathic pain by accumulating effects of noradrenaline in the spinal cord. Anesth Analg 126:298-307.

27. Gao YJ, Zhang L, Ji RR (2010) Spinal injection of TNF-aactivated astrocytes produces persistent pain symptom mechanical allodynia by releasing monocyte chemoattractant protein-1. Glia 58:1871-1880.
28. Gao H, Wu X, Fossett N (2009) Upregulation of the drosophila friend of GATA gene U-shaped by JAK/STAT signaling maintains lymph gland prohemocyte potency. Mol Cell Biol 29:6086-6096.

29. Shin J, Yin Y, Kim DK, Lee SY, Lee W, Kang JW, Kim DW, Hong J (2019) Foxp3 plasmid-encapsulated PLGA nanoparticles attenuate pain behavior in rats with spinal nerve ligation. Nanomedicine (Lond) 18:90-100.

30. Zhang Y, Yu XJ, Chen WS, Gao HL, Liu KL, Shi XL, Fan XY, Jia LL, Cui W, Zhu GQ, Liu JJ, Kang YM (2016) Exercise training attenuates renovascular hypertension partly via RAS- ROSglutamate pathway in the hypothalamic paraventricular nucleus. Sci Rep 6:37467.

31. Thakur M, Rahman W, Hobbs C, Dickenson AH, Bennett DL (2012) Characterisation of a peripheral neuropathic component of the rat monoiodoacetate model of osteoarthritis. PLoS One 7:e33730.

32. Yao L, Kan EM, Lu J, Hao A, Dheen ST, Kaur C, Ling EA (2013) Toll-like receptor 4 mediates microglial activation and production of inflammatory mediators in neonatal rat brain following hypoxia: role of TLR4 in hypoxic microglia. J Neuroinflammation 10:23.

33. Chen Y, Nie H, Tian L, Tong L, Deng J, Zhang Y, Dong H, Xiong L (2015) Sevoflurane preconditioning-induced neuroprotection is associated with Akt activation via carboxyterminal modulator protein inhibition. Br J Anaesth 114:327335.

34. Hochman JR, Gagliese L, Davis AM, Hawker GA (2011) Neuropathic pain symptoms in a community knee OA cohort. Osteoarthritis Cartilage 19:647-654.

35. Oteo-Álvaro Á, Ruiz-Ibán MA, Miguens X, Stern A, Villoria J, Sánchez-Magro I (2015) High prevalence of neuropathic pain features in patients with knee osteoarthritis: a crosssectional study. Pain Pract 15:618-626.

36. Kawai T, Akira S (2007) Signaling to NF-kappaB by Toll-like receptors. Trends Mol Med 13:460-469.

37. Liu HM, Liu XF, Yao JL, Wang CL, Yu Y, Wang R (2006) Utilization of combined chemical modifications to enhance the blood-brain barrier permeability and pharmacological activity of endomorphin-1. J Pharmacol Exp Ther 319:308-316. 